\title{
Travel time and trajectory moments of conservative solutes in three dimensional heterogeneous porous media under mean uniform flow
}

\author{
Xavier Sanchez-Vila ${ }^{\mathrm{a}, *}$, Alberto Guadagnini ${ }^{\mathrm{b}}$ \\ a Department of Geotechnical Engineering and Geosciences, Technical University of Catalonia, Gran Capità S/N, 08034 Barcelona, Spain \\ ${ }^{\mathrm{b}}$ Dipartimento di Ingegneria Idraulica, Ambientale, Infrastrutture Viarie, Rilevamento, Politecnico di Milano, \\ Piazza L. Da Vinci 32, 20133 Milano, Italy
}

Received 26 February 2003; received in revised form 30 June 2004; accepted 30 December 2004

\begin{abstract}
We present expressions satisfied by the first statistical moments (mean and variance-covariance) of travel time and trajectory of conservative solute particles advected in a three-dimensional heterogeneous aquifer under uniform in the mean flow conditions. Closure of the model is obtained by means of a consistent second-order expansion in $\sigma_{Y}$ (standard deviation of the log hydraulic conductivity) of (statistical) moments of quantities of interest. As such, the results obtained are nominally limited to mildly non-uniform fields, with $\sigma_{Y}<1$. Resulting mean and variance of particles travel time and trajectory are functions of first and second moments and cross-moments of trajectory and velocity components. Our solution is applicable to infinite domains and is free of distributional assumptions. As an important application of the methodology we obtain closed-form expressions for the unconditional mean and variance of travel time and particle trajectory for isotropic log-conductivity domain characterized by an exponential variogram. This allows us to recover the non linear behavior of mean travel time versus distance, in agreement with numerical results published in the literature, as well as a non-linear effect in the mean trajectory. The analysis of trajectory variance allows recovering some known results regarding transverse macro-dispersion, evidencing some limitations typical of perturbation theory.
\end{abstract}

(C) 2005 Elsevier Ltd. All rights reserved.

Keywords: Heterogeneous media; Travel time; Particle trajectory; Stochastic analysis; Three-dimensional media

\section{Introduction}

While the physics of groundwater flow and solute transport are known and describable by relatively simple equations, the parameters involved in such equations are extremely variable in space. Thus, the prediction of solute movement in groundwater is highly uncertain. The field of stochastic hydrogeology abandons the idea (typical of deterministic models) of calculating actual flow

\footnotetext{
* Corresponding author.

E-mail address: xavier.sanchez-vila@upc.edu (X. Sanchez-Vila).
}

and transport state variables (hydraulic heads, flow rates, travel times, trajectories,...) and is oriented toward rendering ensemble moments of such quantities. Most of the studies in stochastic hydrogeology have been devoted to find low-order moments. First-order moments constitute unbiased predictors of the variables under study. Second-order moments (variances-covariances) can be interpreted as measures of predictive uncertainty.

There are two main categories of approaches aiming at evaluating the (ensemble) moments of solute trajectories and travel times in random media. The first one aims at obtaining the statistics of particle location at a 
(deterministically) given time, $t$. The second, which we pursue in this work, allows computing the travel time statistics for a particle starting from a given point in space and reaching a given discharge location. In this second approach both the travel time and the particle trajectory are viewed as random variables $[5,8]$.

A summary of the main results available in the literature for the statistical moments of solute travel time and/or trajectory can be found in $[15,16]$. In these works the authors derived a fully non-linear suite of expressions to calculate mean and variance of travel time and trajectory of a conservative solute in two dimensional bounded domains under non-uniform mean flow conditions. In this Technical Note we provide an extension of this work to the three dimensional case, focusing on uniform in the mean flow conditions in unbounded domains. This simplified flow regime allows us presenting closed-form expressions for the mean and variance of travel time and the variance of transverse particle location. The resulting moments are checked against numerical simulations and to the body of analytical solutions already available in the literature.
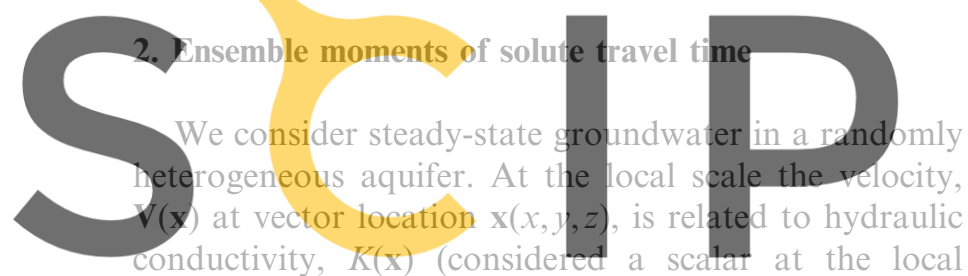

scale), and hydraulic head, $h(\mathbf{x})$ through Darcy's law:

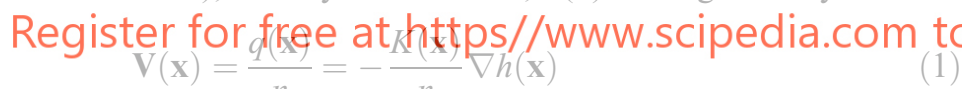

where $q(\mathbf{x})$ is the specific fiux and $n$ is the effective porosity, here taken as constant. The trajectory of a conservative solute in three-dimensional domains is rendered by the kinematic equation:

$$
\begin{aligned}
\mathrm{d} \mathbf{x} & =(\mathrm{d} x, \mathrm{~d} y, \mathrm{~d} z) \\
& =\left(V_{x}(x, y, z) \mathrm{d} t, V_{y}(x, y, z) \mathrm{d} t, V_{z}(x, y, z) \mathrm{d} t\right) \\
& =\mathbf{V}(\mathbf{x}) \mathrm{d} t
\end{aligned}
$$

Here we only consider the advective component of transport and disregard local dispersion. Therefore, all particles injected at a given point within a steady-state flow field follow the same trajectory.

The solution of the coupled system given by (2) renders the position reached at time $t$ by the particle originated from location $\mathbf{x}=\mathbf{x}_{0}$ at time $t=t_{0}$ and is given in parametric form by

$x=x\left(t, t_{0}\right) ; \quad y=y\left(t, t_{0}\right) ; \quad z=z\left(t, t_{0}\right)$

From this point on we will assume that the $x$-coordinate is oriented along the mean flow direction, while $y$ and $z$ are two orthogonal coordinates, transverse to $x$. Upon obtaining $t$ as a function of $x$ from the first of (3), with the assumption that $x=x\left(t, t_{0}\right)$ is invertible, and substituting it into the remaining two equations, we are in a position to write the explicit equation for the trajectory:

$$
\left\{\begin{array}{l}
y=\eta\left(x, \mathbf{x}_{0}\right) \\
z=\zeta\left(x, \mathbf{x}_{0}\right)
\end{array}\right.
$$

Combining (4) and (2) we can write a differential equation for the projection of the trajectory along the $x$-coordinate in terms of the Lagrangian velocity, $V_{x}\left(x, \eta\left(x, \mathbf{x}_{0}\right), \zeta\left(x, \mathbf{x}_{0}\right)\right)$ (in short $\left.V_{x}(x, \eta, \zeta)\right)$, leading to

$\mathrm{d} t=\frac{\mathrm{d} x}{V_{x}(x, \eta, \zeta)}$

The time required for a particle injected (at $t=t_{0}=0$ ) at $\mathbf{x}=\mathbf{x}_{0}$ and traveling along the trajectory to reach a point with coordinate $X_{1}$ (which is the definition of residence or travel time) can be expressed upon integration of (5):

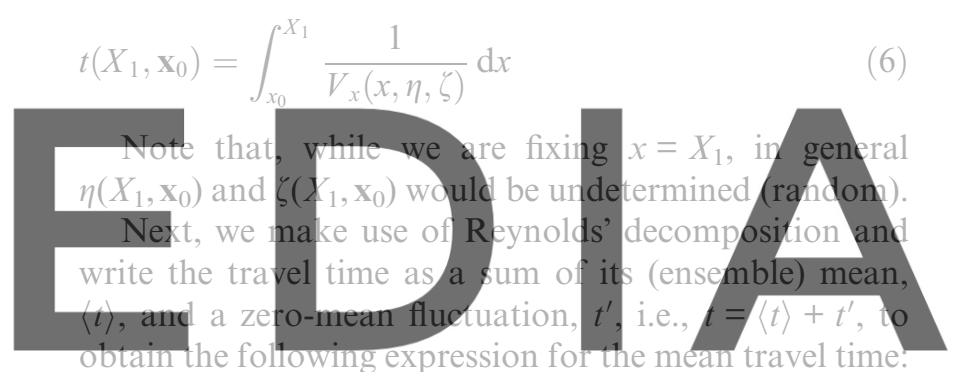

\section{download the $\oint_{x_{0}}^{x_{1}}$ ersion without the watermark}

Following a procedure similar to [16] the travel time variance, $\sigma_{t}^{2}$, is given by

$$
\begin{aligned}
\sigma_{t}^{2}\left(X_{1}, \mathbf{x}_{0}\right) & =\left\langle\left[t^{\prime}\left(X_{1}, \mathbf{x}_{0}\right)\right]^{2}\right\rangle \\
& =\left\langle\left[\int_{x_{0}}^{X_{1}}\left(\frac{1}{V_{x}(x, \eta, \zeta)}-\left\langle\frac{1}{V_{x}(x, \eta, \zeta)}\right\rangle\right) \mathrm{d} x\right]^{2}\right\rangle
\end{aligned}
$$

Eqs. (7) and (8) are expressed in terms of (the heterogeneous functions) $\left\langle 1 / V_{x}\right\rangle$ and $1 / V_{x}$, evaluated along the (random) particle trajectory. They offer the mean and variance of travel time that an ideal solute particle released at $\mathbf{x}_{0}$ takes to reach a given coordinate $X_{1}$, corresponding to (generally random) coordinates $Y_{1}$ and $Z_{1}$ (some exceptions would be, e.g., the case of flow to a single point, where $Y_{1}$ and $Z_{1}$ are deterministically known, or to a vertical draining well of negligible radius, where $Y_{1}$ is deterministic while $Z_{1}$ is random).

To render these expressions workable we applied Reynolds' decomposition to velocity $V_{x}$ and particle transverse displacements, $\eta=\eta\left(x, \mathbf{x}_{0}\right)$ and $\zeta=\zeta\left(x, \mathbf{x}_{0}\right)$, and write them as the sum of their ensemble means, 
$\left\langle V_{x}\right\rangle,\langle\eta\rangle$, and $\langle\zeta\rangle$, and zero-mean fluctuations, $V_{x}^{\prime}, \eta^{\prime}, \zeta^{\prime}$, respectively. This leads to

$$
\begin{aligned}
\frac{1}{V_{x}(x, \eta, \zeta)} & =\frac{1}{\left\langle V_{x}(x, \eta, \zeta)\right\rangle+V_{x}^{\prime}(x, \eta, \zeta)} \\
& =\frac{1}{\left\langle V_{x}(x, \eta, \zeta)\right\rangle}\left[1+\frac{V_{x}^{\prime}(x, \eta, \zeta)}{\left\langle V_{x}(x, \eta, \zeta)\right\rangle}\right]^{-1}
\end{aligned}
$$

Expanding the second factor of (9) in power series, with the assumption that $\left|V_{x}^{\prime}(x, \eta, \zeta)\right| /\left|\left\langle V_{x}(x, \eta, \zeta)\right\rangle\right|<1$, and disregarding terms with powers of fluctuations larger than 2, yields

$\frac{1}{V_{x}(x, \eta, \zeta)} \approx \frac{1}{\left\langle V_{x}(x, \eta, \zeta)\right\rangle}\left[1-\frac{V_{x}^{\prime}(x, \eta, \zeta)}{\left\langle V_{x}(x, \eta, \zeta)\right\rangle}+\frac{V_{x}^{\prime 2}(x, \eta, \zeta)}{\left\langle V_{x}(x, \eta, \zeta)\right\rangle^{2}}\right]$

Expanding $V_{x}(x, \eta, \zeta)$ around its mean trajectory, $[\langle\eta\rangle=\bar{\eta} ;\langle\zeta\rangle=\bar{\zeta})]$, in Taylor's series and disregarding terms with powers of fluctuations larger than two leads to the following expression for the component of velocity fluctuation along $x$-direction (see Appendix A for details about the derivation):

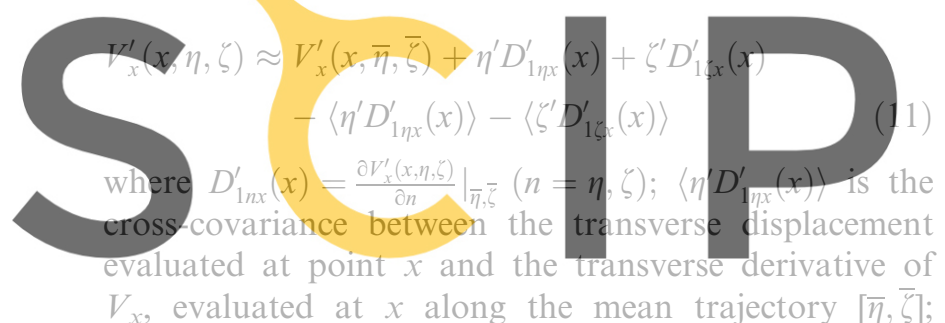

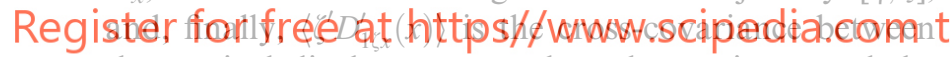

the vertical displacement evaluated at point $x$ and the vertical derivative of $V_{x}$, evaluated at $x$ along the mean trajectory $[\bar{\eta}, \bar{\zeta}]$.

On the other hand, from (11) and dropping higher order terms

$V_{x}^{\prime 2}(x, \eta, \zeta) \approx V_{x}^{\prime 2}(x, \bar{\eta}, \bar{\zeta})$

From (10)-(12), the results presented in Appendix A, and after some expansions, we can write the final expression for the inverse of velocity:

$\frac{1}{V_{x}(x, \eta, \zeta)} \approx \frac{1}{\left\langle V_{x}(x, \bar{\eta}, \bar{\zeta})\right\rangle}\left[1-N_{1}(x)+N_{2}(x)\right]$

where

$$
\begin{aligned}
& N_{1}(x)=\frac{V_{x}^{\prime}(x, \bar{\eta}, \bar{\zeta})+\eta^{\prime} D_{1 \eta x}^{\prime}(x)+\zeta^{\prime} D_{1 \zeta x}^{\prime}(x)}{\left\langle V_{x}(x, \bar{\eta}, \bar{\zeta})\right\rangle} \\
& N_{2}(x)=\frac{V_{x}^{\prime 2}(x, \bar{\eta}, \bar{\zeta})}{\left\langle V_{x}(x, \bar{\eta}, \bar{\zeta})\right\rangle^{2}}
\end{aligned}
$$

Substituting (13) into (6) and disregarding powers of fluctuations larger than 2 leads to an expression for the (random) travel time. Finally, ensemble averaging yields the following expression for the mean travel time:

$$
\begin{aligned}
\left\langle t\left(X_{1}, \mathbf{x}_{0}\right)\right\rangle= & \int_{x_{0}}^{X_{1}} \frac{1}{\left\langle V_{x}(x, \bar{\eta}, \bar{\zeta})\right\rangle}\left[1-\frac{\left\langle\eta^{\prime} D_{1 \eta x}^{\prime}(x)\right\rangle}{\left\langle V_{x}(x, \bar{\eta}, \bar{\zeta})\right\rangle}\right. \\
& \left.-\frac{\left\langle\zeta^{\prime} D_{1 \zeta x}^{\prime}(x)\right\rangle}{\left\langle V_{x}(x, \bar{\eta}, \bar{\zeta})\right\rangle}+\frac{\left\langle V_{x}^{\prime 2}(x, \bar{\eta}, \bar{\zeta})\right\rangle}{\left\langle V_{x}(x, \bar{\eta}, \bar{\zeta})\right\rangle^{2}}\right] \mathrm{d} x
\end{aligned}
$$

where $\left\langle V_{x}^{\prime 2}(x, \bar{\eta}, \bar{\zeta})\right\rangle$ is the variance of the velocity $V_{x}$, evaluated at $x$ along the mean trajectory, $\left[\bar{\eta}\left(x, \mathbf{x}_{0}\right)\right.$; $\left.\bar{\zeta}\left(x, \mathbf{x}_{0}\right)\right]$, of a tracer particle released at $\mathbf{x}_{0}$.

We are now in the position to write the expression of the travel time variance for a particle injected at a location of abscissa $x_{0}$ and ending at a point with abscissa $X_{1}$. After some manipulations and disregarding moments of third-order (and higher), we obtain

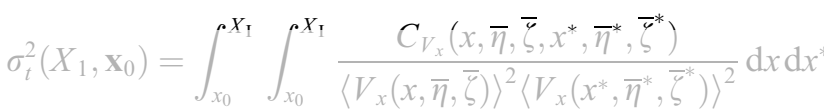

\section{Ensemble moments of particles trajectory}

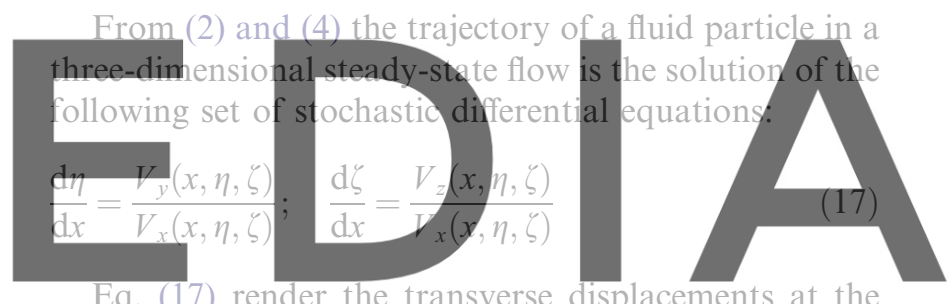

Eq. (17) render the transverse displacements at the generic abscissa $x$ of the particle passing through the

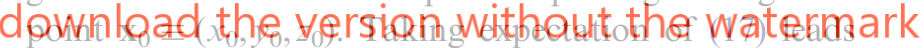

to the following differential equations for the mean trajectory

$\frac{\mathrm{d}\langle\eta\rangle}{\mathrm{d} x}=\left\langle\frac{V_{y}(x, \eta, \zeta)}{V_{x}(x, \eta, \zeta)}\right\rangle ; \quad \frac{\mathrm{d}\langle\zeta\rangle}{\mathrm{d} x}=\left\langle\frac{V_{z}(x, \eta, \zeta)}{V_{x}(x, \eta, \zeta)}\right\rangle$,

subject to the boundary condition

$\left\langle\eta\left(x, \mathbf{x}_{0}\right), \zeta\left(x, \mathbf{x}_{0}\right)\right\rangle=\left\langle y_{0}, z_{0}\right\rangle ; \quad x \equiv x_{0}$,

One should note that in some cases $y_{0}$ and $z_{0}$ are deterministically known and therefore $\left\langle y_{0}\right\rangle=y_{0}$ and $\left\langle z_{0}\right\rangle=z_{0}$. The general expression for the ratio between the velocity components is developed in Appendix D at second-order (in powers of fluctuations). Identifying $j=y$ in (D.4) and taking expectations, we can write the second-order approximation of the equation satisfied by the particle mean transverse displacement, $\left\langle\eta\left(x, \mathbf{x}_{0}\right)\right\rangle$, being

$\frac{\mathrm{d}\left\langle\eta\left(x, \mathbf{x}_{0}\right\rangle\right.}{\mathrm{d} x}=\frac{\left\langle\eta^{\prime} D_{1 \eta \eta y}^{\prime}\right\rangle+\left\langle\zeta^{\prime} D_{1 \zeta \zeta y}^{\prime}\right\rangle}{\left\langle V_{x}(x, \bar{\eta}, \bar{\zeta})\right\rangle}$

where $D_{1 n y}^{\prime}=\left.\frac{\partial V_{y}^{\prime}(x, \eta, \zeta)}{\partial n}\right|_{\bar{\eta}, \bar{\zeta}}(n=\eta, \zeta)$. The equation satisfied by $\frac{\mathrm{d}\langle\zeta\rangle}{\mathrm{d} x}$ is formally similar to the right-hand side of (19), just replacing $V_{y}^{\prime}(x, \bar{\eta}, \bar{\zeta})$ by $V_{z}^{\prime}(x, \bar{\eta}, \bar{\zeta})$. 
Next, we develop an expression for the variancecovariance of the particle trajectory. First, we multiply (17) by the fluctuations of the trajectory at the abscissa $x^{*}, \eta^{*^{\prime}}=\eta^{\prime}\left(x^{*}, \mathbf{x}_{0}\right)$, and $\zeta^{*^{\prime}}=\zeta^{\prime}\left(x^{*}, \mathbf{x}_{0}\right)$, respectively. We then take expectation and obtain the following set of equations:

$$
\begin{aligned}
\left\langle\eta^{* \prime} \frac{\mathrm{d} \eta}{\mathrm{d} x}\right\rangle & =\left\langle\eta^{* \prime} \frac{V_{y}(x, \eta, \zeta)}{V_{x}(x, \eta, \zeta)}\right\rangle \\
\left\langle\zeta^{* \prime} \frac{\mathrm{d} \eta}{\mathrm{d} x}\right\rangle & =\left\langle\zeta^{* \prime} \frac{V_{y}(x, \eta, \zeta)}{V_{x}(x, \eta, \zeta)}\right\rangle \\
\left\langle\eta^{* \prime} \frac{\mathrm{d} \zeta}{\mathrm{d} x}\right\rangle & =\left\langle\eta^{* \prime} \frac{V_{z}(x, \eta, \zeta)}{V_{x}(x, \eta, \zeta)}\right\rangle \\
\left\langle\zeta^{* \prime} \frac{\mathrm{d} \zeta}{\mathrm{d} x}\right\rangle & =\left\langle\zeta^{* \prime \prime} \frac{V_{z}(x, \eta, \zeta)}{V_{x}(x, \eta, \zeta)}\right\rangle
\end{aligned}
$$

Writing the trajectory components as the sum of their ensemble mean and fluctuation and noting that $\eta^{* \prime}$ and $\zeta^{* \prime}$ do not depend on $x$, the equations satisfied by the trajectory covariances, $C_{\eta \eta}\left(x, x^{*}, \mathbf{x}_{0}\right)=\left\langle\eta^{\prime}\left(x, \mathbf{x}_{0}\right) \eta^{\prime}\left(x^{*}, \mathbf{x}_{0}\right)\right\rangle$, $C_{\zeta \zeta}\left(x, x^{*}, \mathbf{x}_{0}\right)=\left\langle\zeta^{\prime}\left(x, \mathbf{x}_{0}\right) \zeta^{\prime}\left(x^{*}, \mathbf{x}_{0}\right)\right\rangle$, and $C_{\eta \zeta}\left(x, x^{*}, \mathbf{x}_{0}\right)=$ $C_{\zeta \eta}\left(x, x^{*}, \mathbf{x}_{0}\right)=\left\langle\eta^{\prime}\left(x, \mathbf{x}_{0}\right) \zeta^{\prime}\left(x^{*}, \mathbf{x}_{0}\right)\right\rangle$, are as follows:

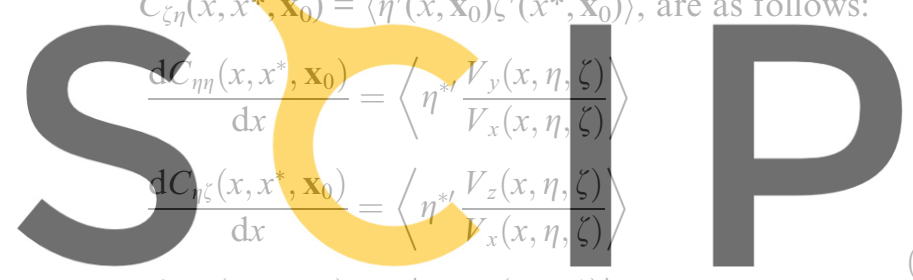

(21)

$\mathrm{d} C_{\check{r n}}\left(x, x^{*}, \mathbf{x}_{0}\right)$

$V_{* * 1} V_{y}(x, \eta, \zeta)$

\section{Register for ${ }^{x}$ free at https $/{ }^{x} / w^{\prime}$ Ww.scipedia.com to}

$$
\frac{\mathrm{d} C_{\zeta \zeta}\left(x, x^{*}, \mathbf{x}_{0}\right)}{\mathrm{d} x}=\left\langle\zeta^{*} \frac{V_{z}(x, \eta, \zeta)}{V_{x}(x, \eta, \zeta)}\right\rangle
$$

with the set of boundary conditions

$$
\begin{aligned}
& C_{\eta \eta}\left(x, x^{*}, \mathbf{x}_{0}\right)=\left\langle y_{0}^{\prime} \eta^{\prime}\left(x^{*}, \mathbf{x}_{0}\right)\right\rangle=C_{\eta \eta}^{0} \\
& C_{\eta \zeta}\left(x, x^{*}, \mathbf{x}_{0}\right)=\left\langle y_{0}^{\prime} \zeta^{\prime}\left(x^{*}, \mathbf{x}_{0}\right)\right\rangle=C_{\eta \zeta}^{0}, \quad x \equiv x_{0} \\
& C_{\zeta \eta}\left(x, x^{*}, \mathbf{x}_{0}\right)=\left\langle z_{0}^{\prime} \eta^{\prime}\left(x^{*}, \mathbf{x}_{0}\right)\right\rangle=C_{\zeta \eta}^{0}, \\
& C_{\zeta \zeta}\left(x, x^{*}, \mathbf{x}_{0}\right)=\left\langle z_{0}^{\prime} \zeta^{\prime}\left(x^{*}, \mathbf{x}_{0}\right)\right\rangle=C_{\zeta \zeta}^{0}
\end{aligned}
$$

If the initial location is deterministically known, then $y_{0}^{\prime}=z_{0}^{\prime}=0$, and thus $C_{\eta \eta}^{0}=C_{\eta \zeta}^{0}=C_{\zeta \eta}^{0}=C_{\zeta \zeta}^{0}=0$.

The next step is to derive analytical expressions for the equations in (21). Discarding terms of third and higher order from (21 top left) and (D.4), we can write, after some manipulations:

$\frac{\mathrm{d} C_{\eta \eta}\left(x, x^{*}, \mathbf{x}_{0}\right)}{\mathrm{d} x}=\frac{C_{\eta V_{y}}\left(x^{*}, x, \mathbf{x}_{0}\right)}{\left\langle V_{x}(x, \bar{\eta}, \bar{\zeta})\right\rangle}$

where $C_{\eta V_{y}}\left(x^{*}, x, \mathbf{x}_{0}\right)=\left\langle\eta^{* \prime} V_{y}^{\prime}(x, \bar{\eta}, \bar{\zeta})\right.$ is the cross-covariance between $\eta^{*}$ and $V_{y}(x, \bar{\eta}, \bar{\zeta})$.
Similarly, the equations satisfied by $C_{\eta \zeta}\left(x, x^{*}, \mathbf{x}_{0}\right)$, $C_{\zeta \eta}\left(x, x^{*}, \mathbf{x}_{0}\right)$, and $C_{\zeta \zeta}\left(x, x^{*}, \mathbf{x}_{0}\right)$ are

$\frac{\mathrm{d} C_{\eta \zeta}\left(x, x^{*}, \mathbf{x}_{0}\right)}{\mathrm{d} x}=\frac{C_{\zeta V_{y}}\left(x^{*}, x, \mathbf{x}_{0}\right)}{\left\langle V_{x}(x, \bar{\eta}, \bar{\zeta})\right\rangle}$

$\frac{\mathrm{d} C_{\zeta \eta}\left(x, x^{*}, \mathbf{x}_{0}\right)}{\mathrm{d} x}=\frac{C_{\eta V_{z}}\left(x^{*}, x, \mathbf{x}_{0}\right)}{\left\langle V_{x}(x, \bar{\eta}, \bar{\zeta})\right\rangle}$

$\frac{\mathrm{d} C_{\zeta \zeta}\left(x, x^{*}, \mathbf{x}_{0}\right)}{\mathrm{d} x}=\frac{C_{\zeta V_{z}}\left(x^{*}, x, \mathbf{x}_{0}\right)}{\left\langle V_{x}(x, \bar{\eta}, \bar{\zeta})\right\rangle}$

with $C_{\zeta V_{j}}\left(x^{*}, x, \mathbf{x}_{0}\right)=\left\langle\zeta^{* \prime} V_{j}^{\prime}(x, \bar{\eta}, \bar{\zeta})\right\rangle(j=x, y, z)$. The corresponding trajectory variances are obtained from the covariances by taking the limit for $x^{*} \rightarrow x$.

These relationships allow predicting the trajectory of a particle starting from a given injection point and reaching a given location of interest, characterized by the abscissa $\left(x=X_{1}\right)$ and (generally random) transverse coordinates $\left(y=Y_{1}, z=Z_{1}\right)$. They also yield a measure of the uncertainty associated to such a predictor.

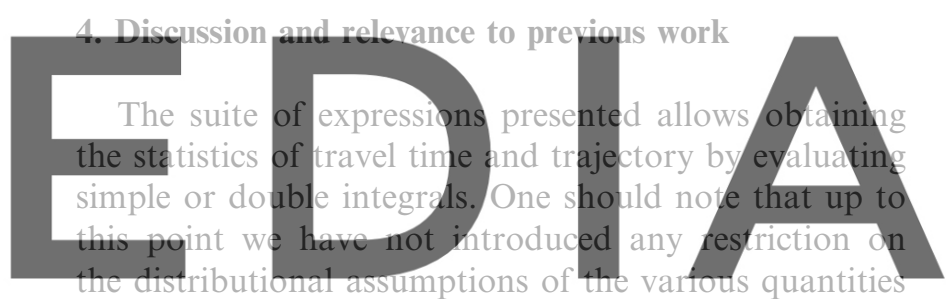

involved (in particular, the equations are not restricted

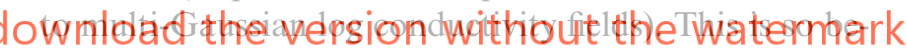
cause they essentially rely on (cross-)moments of velocity which can be easily computed via an extension to three-dimensional space of the method proposed by $[13,14,17]$. Moreover, our approach can be completely integrated with the composite medium approach which allows assessing validity of perturbation approximations when dealing with highly heterogeneous material distributions $[24,25]$. It is also compatible with models treating aquifers as multi-modal heterogeneous formations [18].

In this section we review some of the results that can be found in the literature, while in the next one we particularize the problem to the case of a multi-log Gaussian distribution of $Y=\ln K$, with a given variogram, where analytical closed-form solutions can be obtained.

Finding the moments of travel time and/or particle trajectory in a three dimensional heterogeneous medium has been the subject of the work by several authors. In almost all cases moments are computed for given predefined mean flow conditions. The statistical moments of solute travel time in an infinite domain under uniform mean flow were initially studied by [23] and then extended by $[5,10]$. The simplifying hypothesis used by these authors is to substitute the actual velocity at a 
given point by its projection along the mean trajectory. A direct application of small perturbations for this problem leads to mean travel time becoming linear with travel distance. Numerical simulations in two-dimensions carried out by [6] show that there is a correction term close to the source that leads to a non-linear effect. In order to tackle this non-linearity at short distances, these authors provide an empirical relationship for the transition from near the source to far from the source behaviors. It was then shown [11] that the empirical expression proposed by [6] predicts the mean arrival time in three-dimensional simulations as well when particles are injected evenly in space. When, contrariwise, particles' injection is proportional to flow, mean travel time becomes linear with distance.

The impact of a finite flow domain size on solute travel time statistics for a non-reactive solute in a threedimensional statistically isotropic porous medium under uniform mean flow conditions has been analyzed by [4] within a first-order framework

The only works we are aware in which the authors do not project the velocity along the mean trajectory are those of [21] and [26]. In [21], the author provides an

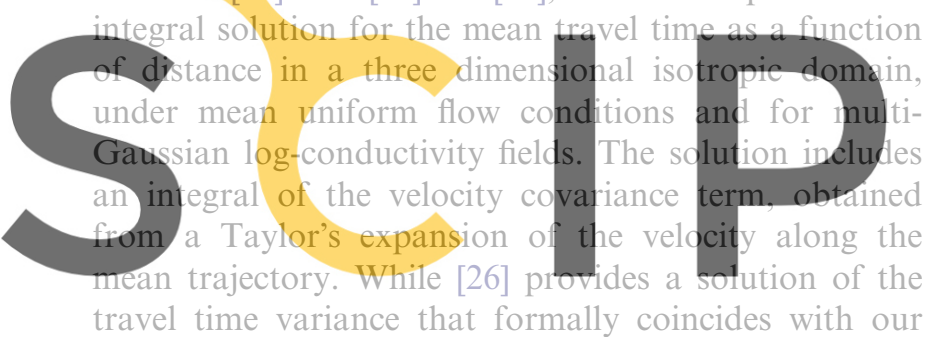

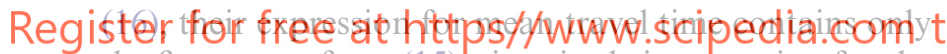
the first term of our (15), since in their expansion for the velocity they drop second-order terms (see also Appendix A for a comment on this point).

With regard to the analysis of trajectories in heterogeneous media, Dagan [7-9] considers a mean uniform flow in an infinite domain of stationary log-conductivity and notes that the trajectory has a normal probability density function (pdf) distribution. For this flow configuration Dagan [7,9] derived closed-form expressions for longitudinal and transverse displacement variance within the classical Lagrangian framework relying upon spectral techniques for representation of velocity (cross-)moments. The assumption of stationarity is basic to the spectral analysis of random flows [3,12], since, in general, it is required for Fourier representation of random fields, such as hydraulic conductivity and hydraulic head. Furthermore, since the presence of boundary conditions renders hydraulic head statistically inhomogeneous, the spectral approach is strictly limited to infinite domains and homogeneous initial conditions. This limitation can be relaxed in some special cases by employing the so-called local stationarity hypothesis [19].

Comparison between the expression of the mean trajectory as obtained by [26] and our solution (19) evi- dences that both coincide at first-order. The obvious result is that the mean trajectory of a particle starting from $\left(x_{0}, y_{0}, z_{0}\right)$, is always $\left(x, y_{0}, z_{0}\right)$. If, at a given time, the particle is (randomly) located at $(y, z) \neq\left(y_{0}, z_{0}\right)$, it will eventually tend to revert (in the mean) towards $(y, z)=\left(y_{0}, z_{0}\right)$. This important feature cannot be grasped by a first-order analysis, while is highlighted by our Eq. (19).

\section{Closed-form solution for multi-Gaussian log- $k$ field with isotropic exponential variogram}

In this section we present a direct application of our procedure. This will be used to check the validity of the approach by recovering some results from the literature and to present some new, closed-form, analytical solutions.

We consider mean uniform flow within an infinite stationary field with simple exponential variogram of the natural logarithm of hydraulic conductivity, $Y=\ln K$, with integral scale $I$. Without losing generality we consider injection at $\mathbf{x}_{0}=0$, while the discharge surface is

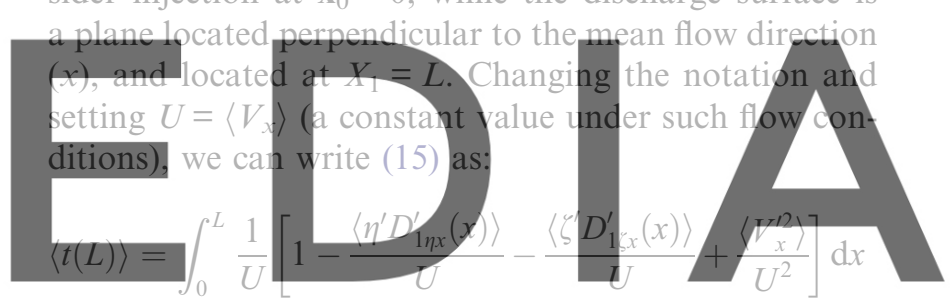

(27)

download the version without the watermark mean velocity, $U$, is given by [9]

$U=\frac{K_{G} \exp \left(\sigma_{Y}^{2} / 6\right) J}{n}$

$K_{G}$ being the geometric mean of hydraulic conductivity, and $J$ the mean gradient. The expression for the velocity variance, $\left\langle V_{x}^{\prime 2}\right\rangle$, that appears in (27) is constant throughout the aquifer and can be obtained from [22]. Expressions for the cross-moments between trajectory fluctuations and derivatives of velocities appearing in (27) are obtained on the basis of the expressions of velocity cross-covariances provided by [22].

After performing the corresponding integration, (27) becomes (up to second-order in $\sigma_{Y}$ ):

$$
\begin{aligned}
\langle t(L)\rangle=\frac{1}{U}[ & L+\sigma_{Y}^{2} I\left(1+\frac{24}{L_{1}^{4}}\left(1-\exp \left(-L_{1}\right)\right)\right. \\
& \left.\left.\quad-\frac{24}{L_{1}^{3}} \exp \left(-L_{1}\right)-\frac{8}{L_{1}^{2}} \exp \left(-L_{1}\right)-\frac{4}{L_{1}^{2}}\right)\right]
\end{aligned}
$$

with $L_{1}=\frac{L}{I}$. Fig. 1 depicts the dependence of mean travel time on distance for different values of $\sigma_{Y}^{2}$. The following 


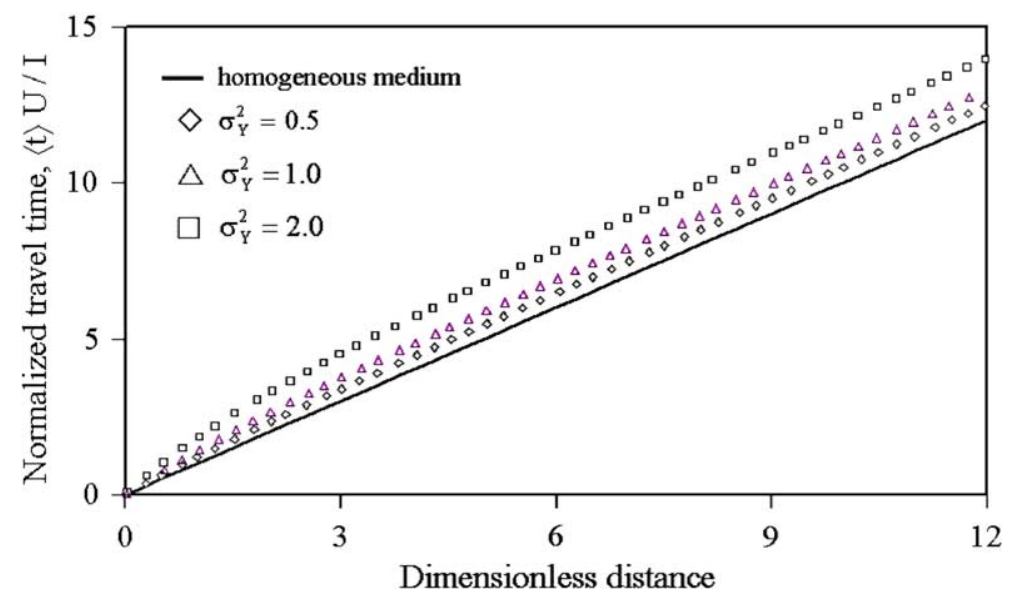

Fig. 1. Normalized mean travel time as a function of dimensionless travel distance for three values of 1 og-conductivity variance $\sigma_{Y}^{2}$ (from Eq. 29)

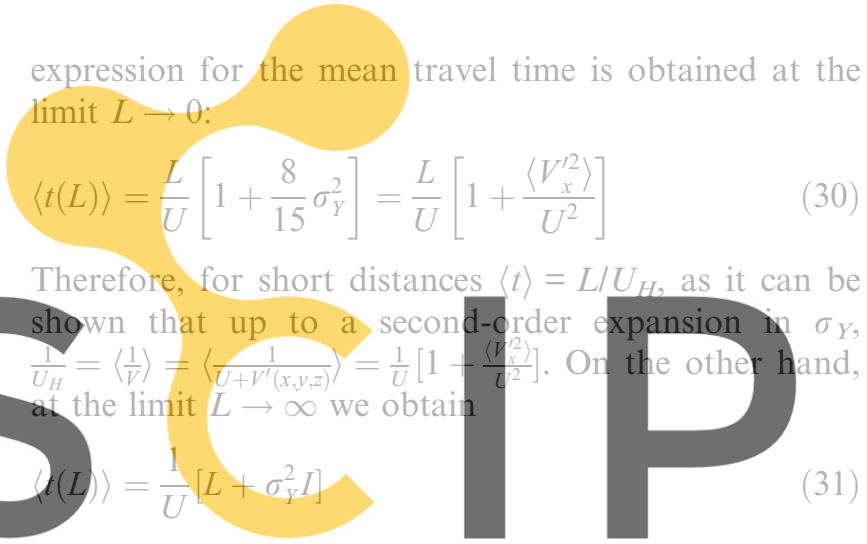

where the leading term is inversely proportional to the arithmetic mean of velocity, Previous results.in the liter-

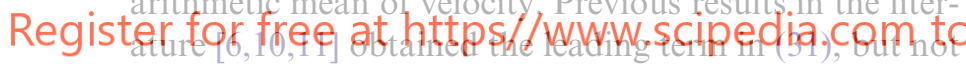
the offset term, which includes the effect of heterogeneity at large distance. There is a clear physical explanation for this behavior. At short distances travel time is inversely proportional to local velocity at the injection point. Therefore, the expected value is proportional to the inverse of the harmonic mean of velocity. On the other hand, for large distances the particles locations are not correlated with the velocity at the initial location. Thus, particles tend to find the fastest paths and transport is speeded. This effect is more important as the degree of heterogeneity increases. We emphasize that the closedform expression (29) describing the nonlinear dependence of mean travel time on distance is an original result which cannot be obtained on the basis of a first-order formalism of the kind proposed by [1] and [26], where only a linear dependence on distance can be recovered.

Now we analyze the variance of travel time. From (16), we can write

$$
\begin{aligned}
\sigma_{t}^{2}\left(L, \mathbf{x}_{0}\right) & =\int_{x_{0}}^{L} \int_{x_{0}}^{L} \frac{\left\langle V_{x}^{\prime}(x, 0,0) V_{x}^{\prime}\left(x^{*}, 0,0\right)\right\rangle}{\left\langle V_{x}(x, 0,0)\right\rangle^{2}\left\langle V_{x}\left(x^{*}, 0,0\right)\right\rangle^{2}} \mathrm{~d} x \mathrm{~d} x^{*} \\
& =\frac{1}{U^{4}} \int_{x_{0}}^{L} \int_{x_{0}}^{L} C_{V_{X}}\left(x-x^{*}, 0,0\right) \mathrm{d} x \mathrm{~d} x^{*}
\end{aligned}
$$

which is the format already provided by [5] (their equation 15). Performing the integration leads to

$$
\sigma_{t}^{2}\left(L, \mathbf{x}_{0}\right)=\frac{\sigma_{Y}^{2} I^{2}}{U^{2}}\left[2 L_{1}-\frac{16}{3}-\frac{16}{L_{1}^{3}}\left(1-\exp \left(-L_{1}\right)\right)\right.
$$

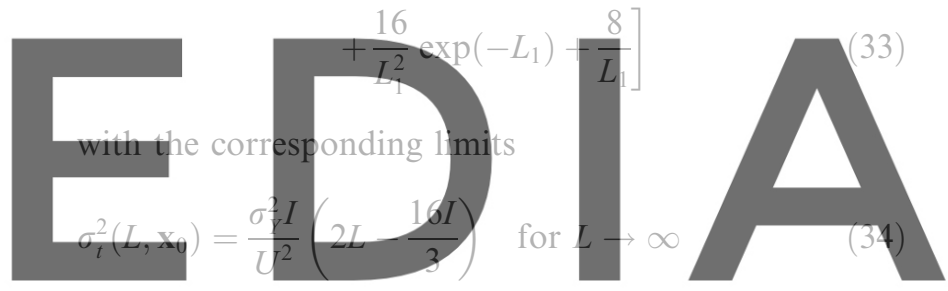

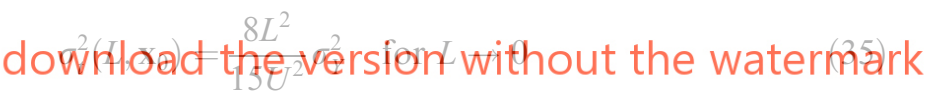

Expression (35) and the leading term in (34) were already found by [23].

As an important application, we note that solute flux statistics (mean and variance) can be expressed in terms of the probability density functions of particle travel time and transverse displacement [1]. Thus, contemplating the effect of the nonlinear dependence evidenced by (29) and making use of the closed-form solution (33) for travel time variance could be employed within the context of the work of [1] to lead to a higher order correction of solute flux moments.

In Fig. 2 we plot travel time variance as a function of travel distance. After a travel distance of 15 integral scales, the error induced by using the asymptotic value (34) rather than the actual value (33) is less than $2 \%$. On the other hand, disregarding the offset term in (34) would entail an error close to $18 \%$ for the same travel distance (the relative error would also decrease with travel distance, but it would need over 130 integral scales to get down to $2 \%$ ).

With reference to the trajectory moments, under uniform mean flow conditions in an infinite domain, equation (19) leads to the obvious result $\left\langle\eta\left(x, \mathbf{x}_{0}\right)\right\rangle=y_{0}$, 


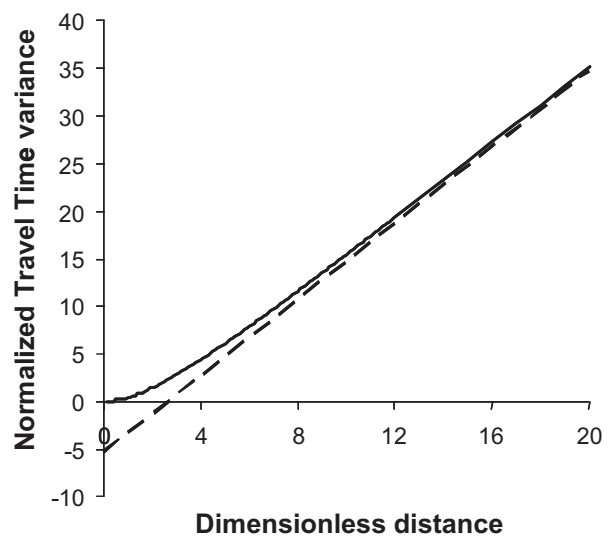

Fig. 2. Normalized travel time variance (defined as $\sigma_{t}^{2} U^{2} / \sigma_{Y}^{2} I^{2}$ ) as a function of dimensionless travel distance $(L / I)$ (from Eq. 33) and the corresponding asymptote.

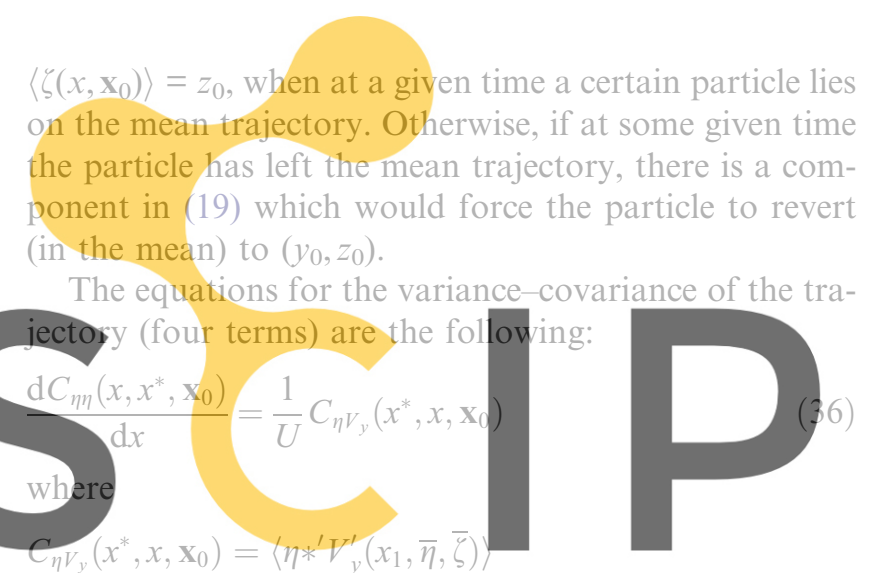

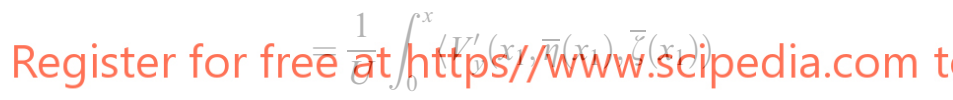

$$
\left.\times V_{y}^{\prime}(x, \bar{\eta}(x), \bar{\zeta}(x))\right\rangle \mathrm{d} x_{1}
$$

Thus

$$
\begin{aligned}
C_{\eta \eta}\left(x^{*}, x, \mathbf{x}_{0}\right)= & \frac{1}{U^{2}} \int_{0}^{x} \int_{0}^{x^{*}}\left\langle V_{y}^{\prime}\left(x_{1}, \bar{\eta}\left(x_{1}\right), \bar{\zeta}\left(x_{1}\right)\right)\right. \\
& \left.\times V_{y}^{\prime}\left(x_{2}, \bar{\eta}\left(x_{2}\right), \bar{\zeta}\left(x_{2}\right)\right)\right\rangle \mathrm{d} x_{1} \mathrm{~d} x_{2}
\end{aligned}
$$

and

$$
\begin{aligned}
\sigma_{\eta \eta}^{2}\left(x, \mathbf{x}_{0}\right)= & \frac{1}{U^{2}} \int_{0}^{x} \int_{0}^{x}\left\langle V_{y}^{\prime}\left(x_{1}, \bar{\eta}\left(x_{1}\right), \bar{\zeta}\left(x_{1}\right)\right)\right. \\
& \left.\times V_{y}^{\prime}\left(x_{2}, \bar{\eta}\left(x_{2}\right), \bar{\zeta}\left(x_{2}\right)\right)\right\rangle \mathrm{d} x_{1} \mathrm{~d} x_{2}
\end{aligned}
$$

where $\bar{\eta}\left(x_{1}\right) \equiv \bar{\eta}\left(x_{2}\right)$ and $\bar{\zeta}\left(x_{1}\right) \equiv \bar{\zeta}\left(x_{2}\right)$. Integration of (39) leads to

$\sigma_{\eta \eta}^{2}\left(L, \mathbf{x}_{0}\right)=2 \sigma_{Y}^{2} I^{2}\left[\frac{1}{3}-\frac{1}{L_{1}}+\frac{4}{L_{1}^{3}}-\exp \left(-L_{1}\right)\left(\frac{4}{L_{1}^{3}}+\frac{4}{L_{1}^{2}}+\frac{1}{L_{1}}\right)\right]$

which is equal to (4.6.15) of [9], obtained by means of spectral analysis. Limiting values are recovered as

$\sigma_{\eta \eta}^{2}\left(L, \mathbf{x}_{0}\right)=\frac{1}{15} \sigma_{Y}^{2} L^{2} \quad$ for $L \rightarrow 0$ $\sigma_{\eta \eta}^{2}\left(L, \mathbf{x}_{0}\right)=\frac{2}{3} \sigma_{Y}^{2} I^{2} \quad$ for $L \rightarrow \infty$

Fig. 3 is a plot of the variance of the transverse component of the trajectory. Notice that, while it reaches a plateau for large travel distance, the particle needs to travel over 60 integral scales to reach $95 \%$ of this limiting value. Fig. 4 depicts the derivative of the variance of transverse particle displacement with respect to distance. This value is usually related to the concept of macrodispersion [9]. The limiting values for the derivative of the transverse component are zero for both $L \rightarrow 0$ and $L \rightarrow \infty$, thus suggesting that transverse dispersion in three-dimensions tends to vanish for large travel distances. However, one should be aware that this specific result suffers from limitations typical of the perturbation theory used. As was first suggested by Neuman and Zhang $[20,27]$ and recently demonstrated analytically

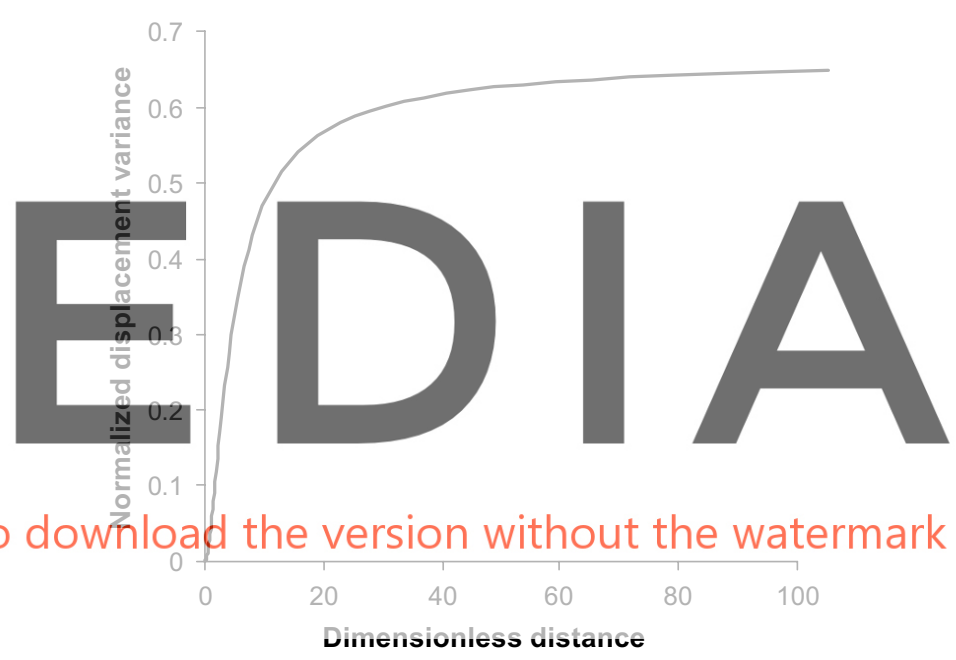

Fig. 3. Variance of the normalized transversal component of the trajectory $\left(\sigma_{\eta \eta}^{2} / \sigma_{Y}^{2} I^{2}\right)$ as a function of dimensionless distance.

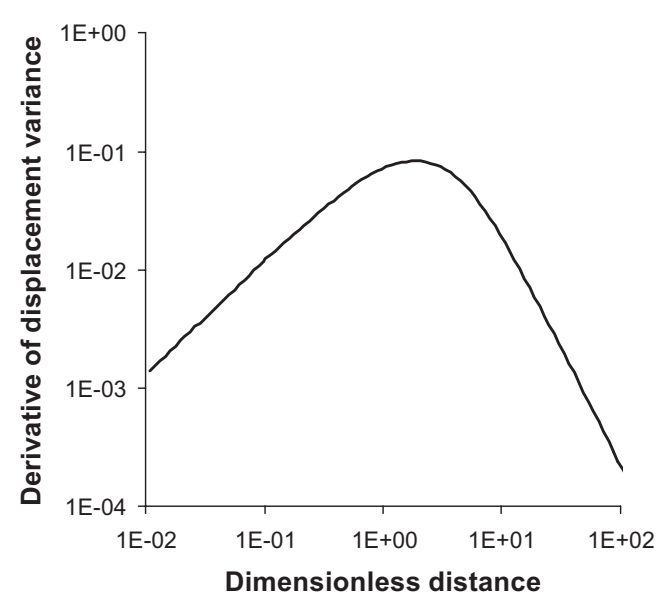

Fig. 4. Derivative of the variance of the normalized transversal component of the trajectory $\left(\frac{1}{\sigma_{Y}^{2} I} \frac{\mathrm{d} \sigma_{\eta}^{2}}{\mathrm{~d} L}\right)$ as a function of dimensionless distance. 
[2], higher order contributions do not cancel out, yielding finite transverse macrodispersion coefficients in three dimensions.

The cross-variance $C_{\eta \zeta}\left(x, x^{*}, \mathbf{x}_{0}\right)=C_{\zeta \eta}\left(x, x^{*}, \mathbf{x}_{0}\right)$ is then given by

$$
\begin{aligned}
C_{\eta \zeta}\left(x^{*}, x, \mathbf{x}_{0}\right)= & \frac{1}{U^{2}} \int_{0}^{x} \int_{0}^{x^{*}}\left\langle V_{z}^{\prime}\left(x_{1}, \bar{\eta}\left(x_{1}\right), \bar{\zeta}\left(x_{1}\right)\right)\right. \\
& \left.\times V_{y}^{\prime}\left(x_{2}, \bar{\eta}\left(x_{2}\right), \bar{\zeta}\left(x_{2}\right)\right)\right\rangle \mathrm{d} x_{1} \mathrm{~d} x_{2}
\end{aligned}
$$

From [22] the cross-covariance of transversal velocities is 0 along any given line, so $C_{\eta \zeta}\left(x^{*}, x, \mathbf{x}_{0}\right)=0$. Finally, for isotropic correlation $C_{\zeta \zeta}\left(x, x^{*}, \mathbf{x}_{0}\right)=C_{\eta \eta}\left(x, x^{*}, \mathbf{x}_{0}\right)$.

\section{Conclusions}

Our work leads to the following major conclusions.

We have presented second-order expressions for the predictor of travel time and trajectory of conservative solute particles advected in randomly heterogeneous three-dimensional infinite aquifers under uniform in the mean flow conditions. These are complemented

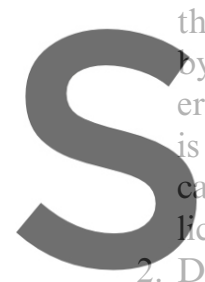
expressions yielding errors. Our solutions rely o free of distributional as ble to either Gaussian o conductivity fields.

Direct application of the resulting expressions to a multi-Gaussian log hydraulic conductivity field with

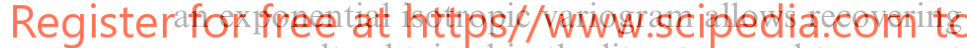
some results obtained in the literature and to present some new closed-form analytical solutions. In particular, it is possible to recover the non-linear dependence of the mean travel time on distance that has been observed in numerical simulations. Mean travel time is inversely proportional to the harmonic mean of velocity for short travel distances. For very large travel distances, mean travel time is given by the sum of (a) a term which increases with travel distance and is proportional to the inverse of the arithmetic mean of velocity, and (b) a constant term (offset) which is a function of the parameters characterizing the heterogeneity.

3. A closed-form expression for the travel time variance is also presented. The expression recovers the limiting value for short travel distances already available in the literature, and provides an additional constant term to the linear limiting value published previously. The closed-form solutions provided for travel time moments could be used to obtain higher order approximations to the solute flux approach methodology developed by [1].

4. The equations satisfied by the mean and covariance of particle trajectories are presented. The former allows us to highlight a non-linear feature of the mean trajectory conditioned to the initial position of the particle. Regarding the latter, when applied to the particular case of a multi-Gaussian log-conductivity field, the resulting expressions agree with those already available in the literature. When interpreting the large scale behavior of second-order trajectory moment and attempting to link it to the concept of transverse macro-dispersion coefficients, one should be aware that this specific result suffers from limitations typical of the perturbation theory.

\section{Acknowledgements}

This work was supported by the European Commission under Contract No. EVK1-CT-1999-00041 (W-SAHARA-Stochastic Analysis of Well Head Protection and Risk Assessment). The first author acknowledges support by ENRESA and the Department d'Universitats, Recerca i Societat de la Informació of the Catalan Government. We thank Shlomo P. Neuman for providing fruitful comments to improve the quality of the

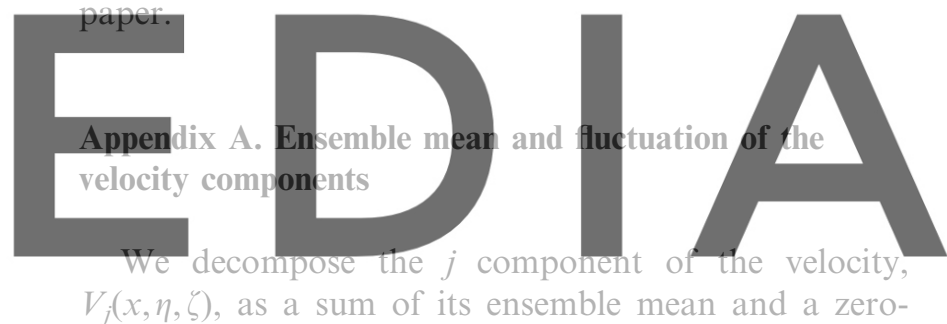

dowaratoadtutamersion without the watermark

$V_{j}(x, \eta, \zeta)=\left\langle V_{j}(x, \eta, \zeta)\right\rangle+V_{j}^{\prime}(x, \eta, \zeta)$

with $\left\langle V_{j}^{\prime}(x, \eta, \zeta)\right\rangle=0$

Expanding $V_{j}(x, \eta, \zeta)$ in a Taylor series around the mean trajectory, $[\bar{\eta}, \bar{\zeta}]$, and disregarding the terms with power of trajectory fluctuations larger than 2, yields

$$
\begin{aligned}
V_{j}(x, \eta, \zeta) \cong & V_{j}(x, \bar{\eta}, \bar{\zeta})+\left.\eta^{\prime} \frac{\partial V_{j}(x, \eta, \zeta)}{\partial \eta}\right|_{\substack{\eta=\bar{\eta} \\
\zeta=\bar{\zeta}}} \\
& +\left.\zeta^{\prime} \frac{\partial V_{j}(x, \eta, \zeta)}{\partial \zeta}\right|_{\substack{\eta=\bar{\eta} \\
\zeta=\bar{\zeta}}}+\left.\frac{\eta^{\prime 2}}{2} \frac{\partial^{2} V_{j}(x, \eta, \zeta)}{\partial \eta^{2}}\right|_{\substack{\eta=\bar{\eta} \\
\zeta=\bar{\zeta}}} \\
& +\left.\frac{\zeta^{\prime 2}}{2} \frac{\partial^{2} V_{j}(x, \eta, \zeta)}{\partial \zeta^{2}}\right|_{\substack{\eta=\bar{\eta} \\
\zeta=\bar{\zeta}}}+\left.\eta^{\prime} \zeta^{\prime} \frac{\partial^{2} V_{j}(x, \eta, \zeta)}{\partial \eta \partial \zeta}\right|_{\substack{\eta=\bar{\eta} \\
\zeta=\bar{\zeta}}}
\end{aligned}
$$

Combining (A.1) and (A.2), disregarding terms with powers of velocity and trajectory fluctuations larger than 2 , and setting for brevity of notation $\left.\right|_{\bar{\eta}, \bar{\zeta}}$ instead of $\left.\right|_{\eta=\bar{\eta}}$, we obtain: 


$$
\begin{aligned}
V_{j}(x, \eta, \zeta) \cong & \left\langle V_{j}(x, \bar{\eta}, \bar{\zeta})\right\rangle+V_{j}^{\prime}(x, \bar{\eta}, \bar{\zeta}) \\
& +\eta^{\prime}\left[\left.\frac{\partial\left\langle V_{j}(x, \eta, \zeta)\right\rangle}{\partial \eta}\right|_{\bar{\eta}, \bar{\zeta}}+\left.\frac{\partial V_{j}^{\prime}(x, \eta, \zeta)}{\partial \eta}\right|_{\bar{\eta}, \bar{\zeta}}\right] \\
& +\zeta^{\prime}\left[\left.\frac{\partial\left\langle V_{j}(x, \eta, \zeta)\right\rangle}{\partial \zeta}\right|_{\bar{\eta}, \bar{\zeta}}+\left.\frac{\partial V_{j}^{\prime}(x, \eta, \zeta)}{\partial \zeta}\right|_{\bar{\eta}, \bar{\zeta}}\right] \\
& +\left.\frac{\eta^{\prime 2}}{2} \frac{\partial^{2}\left\langle V_{j}(x, \eta, \zeta)\right\rangle}{\partial \eta^{2}}\right|_{\bar{\eta}, \bar{\zeta}}+\left.\frac{\zeta^{\prime 2}}{2} \frac{\partial^{2}\left\langle V_{j}(x, \eta, \zeta)\right\rangle}{\partial \zeta^{2}}\right|_{\bar{\eta}, \bar{\zeta}} \\
& +\left.\eta^{\prime} \zeta^{\prime} \frac{\partial^{2}\left\langle V_{j}(x, \eta, \zeta)\right\rangle}{\partial \eta \partial \zeta}\right|_{\bar{\eta}, \bar{\zeta}}
\end{aligned}
$$

It is important to notice that our (A.3) includes terms of higher order when compared to Eq. (8) of [26]. This is crucial to capture the non-linear behavior of mean travel time, as demonstrated in Section 5. Under mean uniform flow (A.3) reduces to:

$V_{j}(x, \eta, \zeta) \cong\left\langle V_{j}(x, \bar{\eta}, \bar{\zeta})\right\rangle+V_{j}^{\prime}(x, \bar{\eta}, \bar{\zeta})$

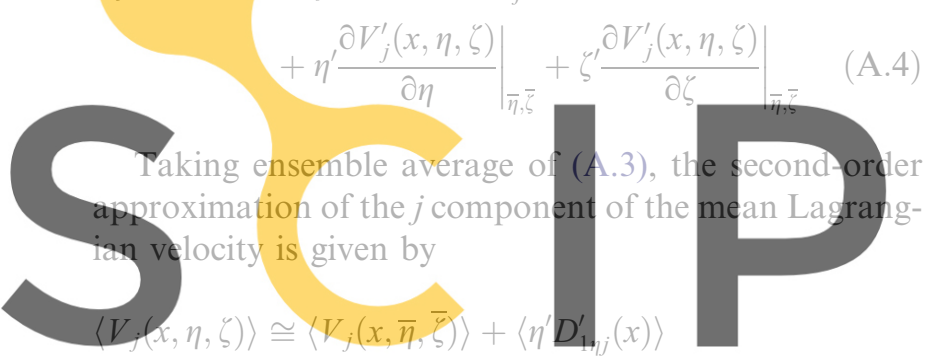

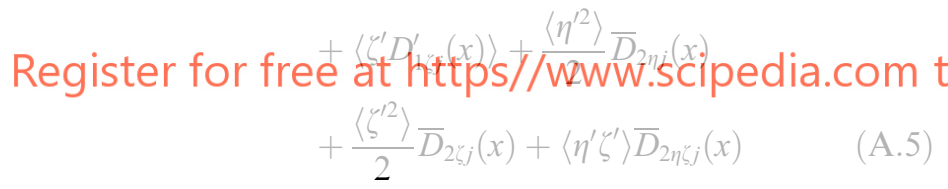

with

$D_{1 n j}^{\prime}(x)=\left.\frac{\partial V_{j}^{\prime}(x, \eta, \zeta)}{\partial n}\right|_{\bar{\eta}, \bar{\zeta}} \quad(n=\eta, \zeta)$

and

$\bar{D}_{2 n j}(x)=\left.\frac{\partial^{2}\left\langle V_{j}(x, \eta, \zeta)\right\rangle}{\partial n^{2}}\right|_{\bar{\eta}, \bar{\zeta}} ; \quad(n=\eta, \zeta)$

$\bar{D}_{2 \eta \zeta j}(x)=\left.\frac{\partial^{2}\left\langle V_{j}(x, \eta, \zeta)\right\rangle}{\partial \eta \partial \zeta}\right|_{\bar{\eta}, \bar{\zeta}}$

In the case of uniform flow in the mean, (A.5) reduces to:

$\left\langle V_{j}(x, \eta, \zeta)\right\rangle \cong\left\langle V_{j}(x, \bar{\eta}, \bar{\zeta})\right\rangle+\left\langle\eta^{\prime} D_{1 \eta j}^{\prime}(x)\right\rangle+\left\langle\zeta^{\prime} D_{1 \zeta j}^{\prime}(x)\right\rangle$

Substituting (A.3) and (A.5) into (A.1) yields the fluctuation component, $V_{j}^{\prime}(x, \eta, \zeta)$ :

$$
\begin{aligned}
V_{j}^{\prime}(x, \eta, \zeta) \cong & V_{j}^{\prime}(x, \bar{\eta}, \bar{\zeta})+\eta^{\prime}\left[\bar{D}_{1 \eta j}(x)+D_{1 \eta j}^{\prime}(x)\right] \\
& +\zeta^{\prime}\left[\bar{D}_{1 \zeta j}(x)+D_{1 \zeta j}^{\prime}(x)\right]+\frac{\bar{D}_{2 \eta j}(x)}{2} \\
& \times\left(\eta^{\prime 2}-\left\langle\eta^{\prime 2}\right\rangle\right)+\frac{\bar{D}_{2 \zeta j}(x)}{2}\left(\zeta^{\prime 2}-\left\langle\zeta^{\prime 2}\right\rangle\right) \\
& +\bar{D}_{2 \eta \zeta j}(x)\left(\eta^{\prime} \zeta^{\prime}-\left\langle\eta^{\prime} \zeta^{\prime}\right\rangle\right) \\
& -\left\langle\eta^{\prime} D_{1 \eta j}^{\prime}(x)\right\rangle-\left\langle\zeta^{\prime} D_{1 \zeta j}^{\prime}(x)\right\rangle
\end{aligned}
$$

which, in the case of mean uniform flow, reduces to (11).

\section{Appendix B. Velocity and trajectory cross-moments}

In this Appendix we derive the equations satisfied by the cross-moment between the trajectory and the $j$ component of the velocity (with $j=x, y, z$ ), i.e. $\left\langle V_{j}^{\prime}\left(x^{*}, \bar{\eta}^{*}, \bar{\zeta}^{*}\right) \eta^{\prime}\right\rangle$ and $\left\langle V_{j}^{\prime}\left(x^{*}, \bar{\eta}^{*}, \bar{\zeta}^{*}\right) \zeta^{\prime}\right\rangle$, where $V_{j}\left(x^{*}, \bar{\eta}^{*}, \bar{\zeta}^{*}\right)$ is the $j$-component of the velocity evaluated along the mean trajectory at the abscissa $x^{*}$. Knowledge of these cross-moments is needed to compute the mean and variance of travel time and trajectory. Multiplying the first of (17) by the random velocity fluctuation $V_{j}^{\prime}\left(x^{*}, \bar{\eta}^{*}, \bar{\zeta}^{*}\right)$

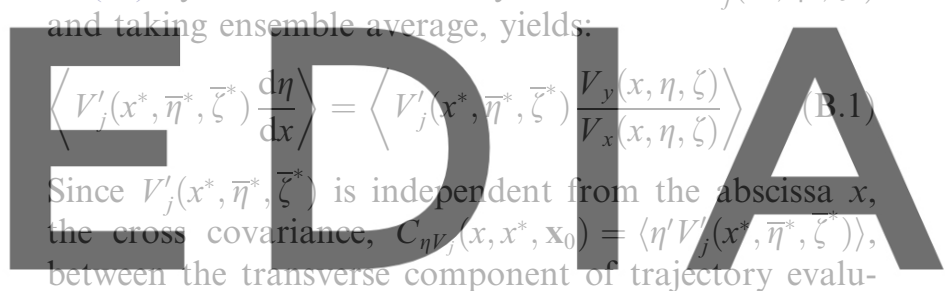

ated at the abscissa $x$ and the j-component of the veloc-

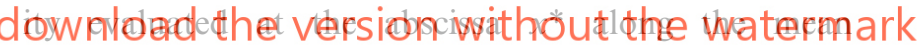
trajectory, satisfies the following equation:

$\frac{\mathrm{d} C_{\eta V_{j}}\left(x, x^{*}, \mathbf{x}_{0}\right)}{\mathrm{d} x}=\left\langle V_{j}^{\prime}\left(x^{*}, \bar{\eta}^{*}, \bar{\zeta}^{*}\right) \frac{V_{y}(x, \eta, \zeta)}{V_{x}(x, \eta, \zeta)}\right\rangle$

subject to the boundary condition

$C_{\eta V_{j}}\left(x, x^{*}, \mathbf{x}_{0}\right)=C_{\eta V_{j}}^{0} \quad x \equiv x_{0}$

where $C_{\eta V_{j}}^{0}=\left\langle y_{0}^{\prime} V_{j}^{\prime}\left(x^{*}, \bar{\eta}^{*}\right)\right\rangle\left(C_{\eta V_{j}}^{0}=0\right.$ would be zero if the position $\mathbf{x}_{0}$ were deterministically known (i.e. $y_{0}^{\prime}=$ $\left.z_{0}^{\prime}=0\right)$ ). Substituting (D.4) into (B.2) and disregarding moments of third-and higher order, the cross-moment between transverse component of trajectory and velocity is the solution of the following differential equation:

$\frac{\mathrm{d} C_{\eta V_{j}}\left(x, x^{*}, \mathbf{x}_{0}\right)}{\mathrm{d} x}=\frac{\left\langle V_{j}^{\prime}\left(x^{*}, \bar{\eta}^{*}, \bar{\zeta}^{*}\right) V_{y}^{\prime}(x, \bar{\eta}, \bar{\zeta})\right\rangle}{\left\langle V_{x}(x, \bar{\eta}, \bar{\zeta})\right\rangle}$

which can be rewritten as

$\frac{\mathrm{d} C_{\eta V_{j}}\left(x, x^{*}, \mathbf{x}_{0}\right)}{\mathrm{d} x}=\frac{C_{V_{j} V_{y}}\left(x^{*}, x, \bar{\eta}^{*}, \bar{\eta}, \bar{\zeta}^{*}, \bar{\zeta}\right)}{\left\langle V_{x}(x, \bar{\eta}, \bar{\zeta})\right\rangle}$

with boundary condition (B.3). Here $C_{V_{j} V_{i}}\left(x^{*}, x, \bar{\eta}^{*}, \bar{\eta}\right.$, $\left.\bar{\zeta}^{*}, \bar{\zeta}\right)=\left\langle V_{j}^{\prime}\left(x^{*}, \bar{\eta}^{*}, \bar{\zeta}^{*}\right) V_{i}^{\prime}(x, \bar{\eta}, \bar{\zeta})\right\rangle$ is the cross-covariance between the components of the velocity along $j$-and 
$i$-directions, evaluated along the mean trajectory at the abscissa $x^{*}$ and $x$, respectively.

The equation satisfied by $C_{\zeta V_{j}}\left(x, x^{*}, \mathbf{x}_{0}\right)=\left\langle\zeta^{\prime} V_{j}^{\prime}\right.$ $\left.\left(x^{*}, \bar{\eta}^{*}, \bar{\zeta}^{*}\right)\right\rangle$ can be obtained upon following a similar procedure as:

$\frac{\mathrm{d} C_{\zeta V_{j}}\left(x, x^{*}, \mathbf{x}_{0}\right)}{\mathrm{d} x}=\frac{C_{V_{j} V_{z}}\left(x^{*}, x, \bar{\eta}^{*}, \bar{\eta}, \bar{\zeta}^{*}, \bar{\zeta}\right)}{\left\langle V_{x}(x, \bar{\eta}, \bar{\zeta})\right\rangle}$

subject to the boundary condition

$C_{\zeta V_{j}}\left(x, x^{*}, \mathbf{x}_{0}\right)=C_{\zeta V_{j}}^{0} \quad x \equiv x_{0}$

The cross-moments at zero lag are obtained upon solving the above system and then taking the limit for $x^{*} \rightarrow x$, as:

(B.8)

Appendix C. Cross-moment between transverse derivative of velocity and trajectory fluctuations

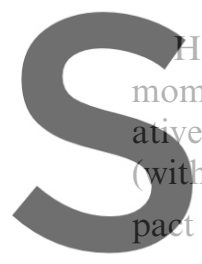
Iere we derive the equat
nent between the trajecto
es of the component of th
h $j=x, y, z)$. These mome
notation as $\left\langle\eta^{\prime} D_{1 n j}^{\prime}\left(x^{*}\right)\right.$
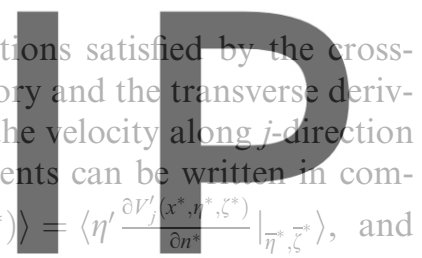

$\left\langle\zeta^{\prime} D_{1 n j}^{\prime}\left(x^{*}\right)\right\rangle=\left\langle\left.\zeta^{\prime} \frac{\partial V_{j}^{\prime}\left(x^{*}, \eta^{*}, \zeta^{*}\right)}{\partial n^{*}}\right|_{\vec{n}^{*}} \vec{\zeta}^{*}\right\rangle$, where $j=x, y, z ;$ and

Registe $\bar{r}$ for free at https//www.scipedia.com to The knowledge of such quantities is crucial to calcuplying (17) by the derivative along direction $n$ of the random velocity fluctuation, and taking expectation yields:

$\left\langle D_{1 n j}^{\prime}\left(x^{*}\right) \frac{\mathrm{d} \eta}{\mathrm{d} x}\right\rangle=\left\langle D_{1 n j}^{\prime}\left(x^{*}\right) \frac{V_{y}(x, \eta, \zeta)}{V_{x}(x, \eta, \zeta)}\right\rangle$

Upon observing that $D_{1 n j}^{\prime}\left(x^{*}\right)$ is independent from the abscissa $x$ and modeling the trajectory as the sum of a mean and a zero-mean random fluctuation, the crosscovariance $C_{\eta D_{1 n j}}\left(x, x^{*}, \mathbf{x}_{0}\right)=\left\langle\eta^{\prime} D_{1 n j}^{\prime}\left(x^{*}\right)\right\rangle$, between the transverse component of trajectory evaluated at the abscissa $x$ and the derivative of the $j$-component of the velocity along direction $n$ evaluated at the abscissa $x^{*}$ along the mean trajectory, satisfies the following equation:

$\frac{\mathrm{d} C_{\eta D_{1 n j}}\left(x, x^{*}, \mathbf{x}_{0}\right)}{\mathrm{d} x}=\left\langle D_{1 n j}^{\prime}\left(x^{*}\right) \frac{V_{y}(x, \eta, \zeta)}{V_{x}(x, \eta, \zeta)}\right\rangle$

with the boundary condition

$C_{\eta D_{1 n j}}\left(x, x^{*}, \mathbf{x}_{0}\right)=C_{\eta D_{1 n j}}^{0} \quad x \equiv x_{0}$ where $C_{\eta D_{1 n j}}^{0}=\left\langle y_{0}^{\prime} D_{1 n j}^{\prime}\left(x^{*}\right)\right\rangle$. Again, $C_{\eta D_{1 n j}}^{0}=0$ if the position $\mathbf{x}_{0}$ is deterministically known (i.e. $y_{0}^{\prime}=z_{0}^{\prime}=0$ ). Substituting (D.4) into (C.2) and disregarding moments of third- and higher order yields

$\frac{\mathrm{d} C_{\eta D_{1 n j}}\left(x, x^{*}, \mathbf{x}_{0}\right)}{\mathrm{d} x}=\left.\frac{1}{\left\langle V_{x}(x, \bar{\eta}, \bar{\zeta})\right\rangle} \frac{\partial C_{V_{j} V_{y}}\left(x^{*}, x, \eta^{*}, \eta, \zeta^{*}, \zeta\right)}{\partial n^{*}}\right|_{\bar{\eta}^{*}, \bar{\zeta}^{*}}$

where $C_{V_{j} V_{k}}$ is the cross-covariance between $j$ - and $i$ components of velocity.

The equation satisfied by $C_{\zeta D_{1 n j}}\left(x, x^{*}, \mathbf{x}_{0}\right)=$ $\left\langle\zeta^{\prime} D_{1 n j}^{\prime}\left(x^{*}\right)\right\rangle=\left\langle\left.\zeta^{\prime} \frac{\partial V_{j}^{\prime}\left(x^{*}, \eta^{*}, \zeta^{*}\right)}{\partial n^{*}}\right|_{\eta^{*}, \bar{\zeta}^{*}}\right\rangle$ can be derived following a similar procedure.

\section{Appendix D. Ratio between velocity vector components}

In this Appendix we develop the expression of the ratio between the $j-(j=y, z)$ and $x$-component of the velocity vector. The $j$-component of the velocity vector is given by (A.1)

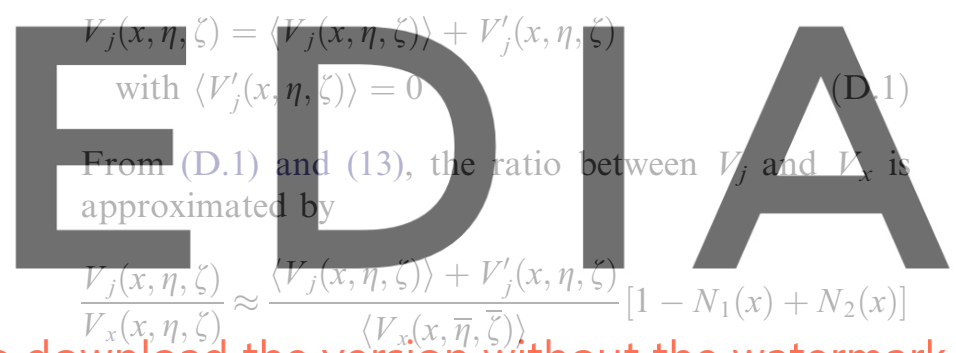

download the version without the watermark

Further disregarding in (D.2) fluctuations of order larger than 2, yields:

$$
\begin{aligned}
\frac{V_{j}(x, \eta, \zeta)}{V_{x}(x, \eta, \zeta)} \cong & \frac{\left\langle V_{j}(x, \eta, \zeta)\right\rangle}{\left\langle V_{x}(x, \bar{\eta}, \bar{\zeta})\right\rangle}\left[1-N_{1}(x)+N_{2}(x)\right] \\
& +\frac{V_{j}^{\prime}(x, \eta, \zeta)}{\left\langle V_{x}(x, \bar{\eta}, \bar{\zeta})\right\rangle}\left[1-\frac{V_{x}^{\prime}(x, \bar{\eta}, \bar{\zeta})}{\left\langle V_{x}(x, \bar{\eta}, \bar{\zeta})\right\rangle}\right]
\end{aligned}
$$

where $N_{1}(x)$ and $N_{2}(x)$ are defined in (14).

Writing the expected value and fluctuation of $V_{j}$ as in (A.4) and (A.6) respectively, and disregarding power of fluctuations larger than 2, (D.3) becomes

$$
\begin{aligned}
\frac{V_{j}(x, \eta, \zeta)}{V_{x}(x, \eta, \zeta)} \cong & \frac{\left\langle V_{j}(x, \bar{\eta}, \bar{\zeta})\right\rangle}{\left\langle V_{x}(x, \bar{\eta}, \bar{\zeta})\right\rangle}\left[1-N_{1}(x)+N_{2}(x)\right] \\
& -\frac{V_{j}^{\prime}(x, \bar{\eta}, \bar{\zeta}) V_{x}^{\prime}(x, \bar{\eta}, \bar{\zeta})}{\left\langle V_{x}(x, \bar{\eta}, \bar{\zeta})\right\rangle^{2}} \\
& +\left[\frac{V_{j}^{\prime}(x, \bar{\eta}, \bar{\zeta})+\eta^{\prime} D_{1 \eta j}^{\prime}(x)+\zeta^{\prime} D_{1 \zeta j}^{\prime}(x)}{\left\langle V_{x}(x, \bar{\eta}, \bar{\zeta})\right\rangle}\right]
\end{aligned}
$$




\section{References}

[1] Andricevic R, Cvetkovic V. Relative dispersion for solute flux in aquifers. J Fluid Mech 1998;361:145-74.

[2] Attinger S, Dentz M, Kinzelbach W. Exact transverse macro dispersion coefficient for transport in heterogeneous porous media. Stochast Environ Res Risk Assessm 2004;18:9-15.

[3] Bakr AA, Gelhar W, Gutjahar L, MacMillan JR. Stochastic analysis of spatial variability in subsurface flows 1 . Comparison of one- and three-dimensional flows. Water Resour Res 1978;14(2): 263-71.

[4] Chang C-M. Uncertainty for solute movement in bounded heterogeneous porous media. Adv Water Res 2003;27:23-30.

[5] Cvetkovic V, Shapiro AM, Dagan G. A solute flux approach to transport in heterogeneous formations 2. Uncertainty analysis. Water Resour Res 1992;28(5):1377-88.

[6] Cvetkovic V, Cheng H, Wen X-H. Analysis of nonlinear effects on tracer migration in heterogeneous aquifers using Lagrangian travel time statistics. Water Resour Res 1996;32(6):1671-80.

[7] Dagan G. Solute transport in heterogeneous porous formations. J Fluid Mech 1984;145:151-77.

[8] Dagan G. Theory of solute transport by groundwater. Ann Rev Fluid Mech 1987;19:183-215.

[9] Dagan G. Flow and transport in porous formations. New York: Springer-Verlag; 1989.

[10] Dagan G, Cvetkovic V, Shapiro AM. A solute flux approach to transport in heterogeneous formations 1 . The general framework. Water Resour Res 1992;28(5):1369-76.

[11] Demmy G, Berglund S, Graham W. Injection mode implications for solute transport in porous media: analysis in a stochastic Lagrangian framework. Water Resour Res 1999;35(7):1965-73.

[12] Gelhar LW. Stochastic subsurface hydrology. New York: Prentice-Hall, Englewood Cliffs; 1993.

[13] Guadagnini A, Neuman SP. Nonlocal and localized analyses of conditional mean steady state flow in bounded, randomly non uniform domains 1 . Theory and computational approach. Water Resour Res 1999;35(10):2999-3018.

[14] Guadagnini A, Neuman SP. Nonlocal and localized analyses of conditional mean steady state flow in bounded, randomly non uniform domains 2. Computational examples. Water Resour Res 1999;35(10):3019-39.

[15] Guadagnini A, Riva M, De Simoni M. Travel time and trajectories of solutes in randomly heterogeneous aquifers. Report
No. D8 of Project "Stochastic Analysis of Well-Head Protection and Risk Assessment-W-SAHaRA", EU Contract EVK1-CT1999-00041, Politecnico di Milano, Department of I.I.A.R., Milano, 2001.

[16] Guadagnini A, Sánchez-Vila X, Riva M, De Simoni M. Mean travel time of conservative solutes in randomly heterogeneous unbounded domains under mean uniform flow. Water Resour Res 2003;39(3): 1050 .

[17] Hernandez AF, Neuman SP, Guadagnini A, Carrera J. Conditioning steady-state mean stochastic flow equations on head and hydraulic conductivity measurements. Stochast Environm Res Risk Assess 2003;17:329-38.

[18] Lu Z, Zhang D. On stochastic modeling of flow in multimodal heterogeneous formations. Water Resour Res 2002;38(10).

[19] Mizell SA, Gutjahr AL, Gelhar LW. Stochastic analysis of spatial variability in two-dimensional steady groundwater flow assuming stationary and nonstationary heads. Water Resour Res 1982; 18(4):1053-67.

[20] Neuman SP, Zhang Y-K. A quasilinear theory of non-Fickian and Fickian subsurface dispersion: 1. Theoretical analysis with application to isotropic media. Water Resour Res 1990;26(5): 887-902.

[21] Rajaram H. Time and scale dependent effective retardation factors in heterogeneous aquifers. Adv Water Res 1997;20(4):217-30.

[22] Rubin Y, Dagan G. A note on head and velocity covariances in three-dimensional flow through heterogeneous anisotropic porous media. Water Resour Res 1992;28(5):1463-70.

[23] Shapiro AM, Cvetkovic VD. Stochastic analysis of solute arrival time in heterogeneous porous media. Water Resour Res 1988; 24(10):1711-8.

[24] Winter CL, Tartakovsky DM. Groundwater flow in heterogeneous composite aquifers. Water Resour Res 2002;38(8): 23.1-23.11.

[25] Winter CL, Tartakovsky DM, Guadagnini A. Numerical solutions of moment equations for flow in heterogeneous composite aquifers. Water Resour Res 2002;38(5):13.1-8.

[26] Wu J, Hu BX, Zhang D, Shirley C. A three-dimensional numerical method of moments for groundwater flow and solute transport in a nonstationary conductivity field. Adv Water Res 2003;26(11):1149-69.

[27] Zhang Y-K, Neuman SP. A quasilinear theory of non-Fickian and Fickian subsurface dispersion: 2. Application to anisotropic media and the borden site. Water Resour Res 1990;26(5):903-13. 